\title{
The longer the reference, the shorter the legs: How response modality affects body perception
}

\author{
Giorgia Tosi ${ }^{1}$ (D) $\cdot$ Daniele Romano ${ }^{1,2}$ \\ Published online: 22 July 2020 \\ (C) The Psychonomic Society, Inc. 2020
}

\begin{abstract}
There is a growing interest in the characterization of the internal body model: a stored representation of the metric properties of the body. Tactile Distance Task (TDT) is an experimental procedure for assessing the body metric, based on the perception of distances between tactile stimuli. We aimed at ruling out potential cognitive confounds: the body part being touched, the response estimation method being used, and the replicability of the results. Crucially, we compared two scoring indices (Global shape index vs. Misestimation of the distance) that have been used in the literature assessing the unique contribution of each score. Our data revealed a distortion of body metric perception of the leg. In particular, we found a more substantial reduction in proximodistal distances rather than in the medio-lateral axis. TDT turned out to be a reliable and replicable method producing consistent results applicable to different body parts. The global shape index was shown to be particularly resistant to contextual experimental factors, while the Misestimation resulted in being affected by the estimation modalities, revealing that the verbal response was the most precise method. Finally, we provided substantial support for the combined use of the two indices as they were shown to give complementary information about body metric representation distortions.
\end{abstract}

Keywords Body metric $\cdot$ Leg representation $\cdot$ Tactile distance perception $\cdot$ Multidimensional scaling $\cdot$ Misestimation

\section{Introduction}

In the last 10 years, there has been a growing interest in the characterization of the body model of size and shape: a stored representation of the metric properties of the body (Longo \& Morcom, 2016; Longo \& Haggard, 2010; Tamè, Braun, Holmes, Farnè, \& Pavani, 2016; Tsakiris, 2010). One of the most used experimental procedures for assessing body metric is based on the perception of distances between distinct tactile stimuli. The rationale behind this procedure is that, in order to

Electronic supplementary material The online version of this article (https://doi.org/10.3758/s13414-020-02074-3) contains supplementary material, which is available to authorized users.

Giorgia Tosi

g.tosi3@campus.unimib.it

Daniele Romano

daniele.romano@unimib.it

1 Department of Psychology, Università degli Studi di Milano Bicocca, Building U6, Piazza dell'Ateneo Nuovo 1, 20126 Milan, Italy

2 NeuroMi - Milan Center for Neuroscience, Milan, Italy estimate the distance between two touches applied on the skin, we need to map those touches on a mental representation of the body part being touched, a stored model that retains the metric properties of the body (Longo, Azañón, \& Haggard, 2010; Longo, Mancini, \& Haggard, 2015; Longo \& Haggard, 2011, Longo \& Haggard, 2012). The tactile distance perception procedure (Longo \& Golubova, 2017) consists of judging the perceived distance between touches applied to the dorsum of a participant's hand by reporting the estimations verbally. In the original proposal of this procedure, a $4 \times 4$ grid of points is marked on the participant's skin, with the rows running along with the medio-lateral hand axis and the columns running along with the proximo-distal one. During testing, two locations are stimulated in sequence by the experimenter, and blindfolded participants make verbal estimates of the perceived distance. The tactile distance perception is used to assess the metric perception of body parts because it relies on both the ability to localize tactile stimulations on the body and to estimate the size of the tactile percept. The perceptual features of the tactile distance perception task make it a promising method in the characterization of metric body representation (Longo, 2015; Tamè, Azañón, \& Longo, 2019). Nevertheless, experimental control focusing on ruling out potential cognitive confounders is still needed. We aimed at studying the 
impact of: (i) the body part being touched, (ii) the response estimation being used, and (iii) the reliability and replicability of the results.

The literature regarding tactile distance perception has mostly focused on the hand (Longo \& Golubova, 2017; Longo et al., 2015; Sadibolova, Tamè, Walsh, \& Longo, 2018). In particular, Longo and co-workers (Longo et al., 2015; Longo \& Morcom, 2016) found that participants' metric perception of the hand is distorted compared to its physical shape, revealing a fat, squat hand representation. We wondered if a similar procedure can be carried out on different body parts (i.e., the legs), less characterized by physical marks like the presence of the fingers or the knuckles that may anchor (or bias) the estimation. Longo and colleagues asked participants to estimate with a verbal response the distances between two touches on the body, asking for an imaginary evaluation based on metric size awareness. During our data collection, a study by Stone, Keizer, and Dijkerman (2018) employed the same experimental procedure on the upper leg.

Interestingly, instead of a verbal estimation, they asked participants to estimate the distance between the tactile stimuli by placing the thumb and the index fingers on a touch screen, reproducing the gap between the touches physically. Such a procedure could not be employed with distances that exceed the hand width. The limitation of non-reproducible lengths could be overcome by providing a visual reference (i.e., a line) for the perceptive recalibration. Nonetheless, a visual stimulus raises additional issues. We questioned if scaling the real distance employing a proportional reproduction would be as precise as providing the copy of the physical length. We also asked if it is better to use an analogic line or a discrete line.

So far, results about the hand and the leg have revealed that people underestimate body distances running along the proximo-distal direction independently of the body district (Longo et al., 2015; Longo \& Morcom, 2016; Stone et al., 2018). Nevertheless, we do not have any information about the replicability of the tactile distance task within the same participant. The experimental question in this latter case asked if the misestimation is stable within a participant and thus if this reflects a perceptual bias that can be assessed multiple times. If so, this measure could be used as a reliable procedure to measure changes in metric body representation that may occur because of specific experimental manipulations, pathological conditions, or interventions.

Notably, although the same distance task was performed on the hand and the legs, the data were processed differently. Longo and collaborators used the Multidimensional Scaling (MDS) and the Procrustes Alignment, two techniques developed for the analysis of perceptual spaces (Friston, Frith, Fletcher, Liddle, \& Frackowiak, 1996), to extract the underlying spatial structure of the tactile perception. MDS assumes that the data represent the distances in an underlying spatial representation (Carroll \& Arabie, 1998): given a matrix of pairwise dissimilarities between items, MDS finds a configuration in which each point represents one of the items, and the distance between them matches the original dissimilarity for all pairs (Cox, 2001). As a method for describing the structure of a system, it has been used with functional neural connectivity (Friston et al., 1996), personality traits (Widiger, Trull, Hurt, Clarkin, \& Frances, 1987), and psychiatric symptoms (Olatunji, Kim, \& Wall, 2015). Longo and Golubova (2017) put together the principal aim of extracting a spatial configuration underlying a set of items and its neuro/psychological applications. They used MDS to construct perceptual maps reflecting body-metric representation as assessed through tactile perception.

Moreover, they compared the perceptual maps obtained with the grid used to perform the task using Procrustes Alignment (PA). PA (Goodall, 1991; Rohlf \& Slice, 1990) superimposes two spatial configurations of homologous landmarks by translating, scaling, and rotating them to be as closely aligned as possible (Longo \& Golubova, 2017). This procedure removes all the non-shape differences (Bookstein, 1991) and provides a dissimilarity index between the two configurations, called the Procrustes Distance. This measure ranges between zero - if two configurations have the same shape, and one - when two structures do not share any spatial structure at all. Taken together, MDS and PA consist of statistical shape analyses and can be used to convey information about the perceived configuration of a set of tactile stimuli.

Instead of using an index of global shape dissimilarity (i.e., Procrustes Distance), Stone et al. (2018) compared the length provided by the participants at each stimuli pair to the actual gap. They calculated the percentage of perceived misestimation using the following equation: $\%$ misestimation $=($ perceived distance - actual distance)/actual distance $* 100$. The Procrustes Distance gives an index of global shape dissimilarity, a quantitative index of how much two shapes are comparable (Longo \& Golubova, 2017). However, it cannot give specific information about the component segments of the shapes. For example, in the case of our squared grid, it cannot tell if the shape distortion is caused by a stretch of the horizontal axes or a contraction of the vertical one (Longo \& Golubova, 2017).

Put differently, the percentage of misestimation provides multiple specific estimation errors that inform about the particular characteristics of the grid, such as the distance between the points or their alignment. Crucially, it does not give a global index of similarity of the entire configurations (Stone et al., 2018). Thus the misestimation index can be informative with regard to directional bias. When the confidence intervals 
(CIs) do not include zero, we can conclude there is a consistent bias, the direction of which is given by the sign of the CIs. The different procedures, in our view, provide complementary non-overlapping information and should be coupled, instead of being used as alternatives.

To better explain the difference between the global shape dissimilarity and the directional bias, we can imagine two different scenarios, given a square shape to be represented (Fig. 1, dashed line). In the first scenario (Fig. 1), two participants represent two rectangular shapes with the same amount of distortion but the opposite sign on the horizontal and the vertical axes. Participant 1 underestimates the horizontal axis $(-50 \%)$ and overestimates the vertical one $(+50 \%)$. On the contrary, participant 2 exceeds the estimations on the horizontal axis $(+50 \%)$ and underestimates the vertical one $(-50 \%)$. Since both participants show the same shape distortion and PA normalizes size (Longo \& Golubova, 2017), the mean Procrustes Distance is higher than zero. On the contrary, the mean percentage of misestimation is 0 on both axes because the directional biases compensate for the two opposite signs.

In the second scenario (Fig. 1), two participants represent two square shapes with different amounts of distortion. Participant 1 overestimates both the horizontal and the vertical axis $(+50 \%$ and $+50 \%$ respectively). On the contrary, participant 2 reproduces the same shape as the real one $(+0 \%$ on both axes). Since the shape is always equal to the model, the mean Procrustes Distance is zero. On the contrary, the mean percentage of misestimation is the average of the different amounts of distortion produced by the participants $(+25 \%$ on both axes). These scenarios show that the Procrustes Distance and the percentage of misestimation convey different types of information about the same task.

This study aimed to consolidate and validate the tactile distance-estimation procedure. The idea is that the Tactile

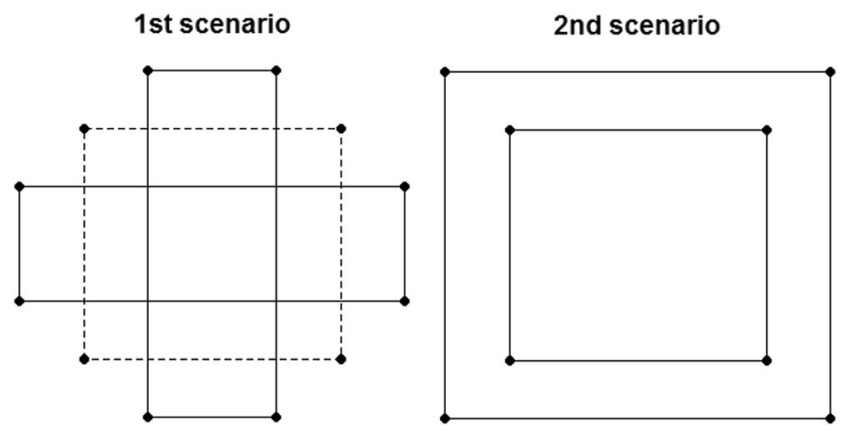

Fig. 1 Examples of scenarios for comparison between the global shape dissimilarity and the direction bias indices. In the first scenario, Directional bias would be 0 , and the global shape dissimilarity would be greater than 0 ; the opposite is exact for the second scenario, where global shape dissimilarity would be blind to the distortion, which can be captured by the Directional bias index
Distance Task (Longo \& Golubova, 2017; Stone, 2018) might be biased by specific cognitive processes that are related to the response modality instead of perceptual bias. Among these potential biases, we can imagine the inability to convert distances in numbers, an anchoring effect due to a previous response, or difficulties in perceptive recalibration. Additionally, the two scoring indices have never been compared directly. We designed a pilot experiment and a subsequent solid experiment to assess this possibility by collecting the estimation with three different modalities (see Methods). Additionally, we analyzed the data by calculating both the Procrustes Distances and the misestimation score, thus providing a robust methodological validation to both the options of collecting the estimates and the way to analyze the bias.

\section{Preliminary experiment}

At the time of data collection, the paper by Stone et al. (2018) was not yet published. Indeed, we wanted to test the feasibility of the tactile distance task with our materials and the body part location selected (i.e., the upper leg). We expected to find a distortion similar to the one that emerged for the hand (Longo \& Golubova, 2017; Longo et al., 2015; Longo $\&$ Morcom, 2016). Publication of the work by Stone et al. (2018) introduced a novel error-estimation procedure (i.e., the percentage of misestimation) to the task. After that, we decided to use both the global shape dissimilarity (i.e., the Procrustes Distance) and the rate of misestimation to analyze our data.

\section{Material and methods}

\section{Participants}

Ten healthy subjects participated in the study (nine female, mean age: $25.50 \pm .85$ years; mean school age: $18.8 \pm .63$ yearas). All participants had normal or corrected-to-normal vision and were naïve to the purpose of the experiment. All participants gave their written informed consent before participating. The study was approved by the local Ethics Committee "Commissione per la Valutazione della Ricerca, Dipartimento di Psicologia" of the University of Milano-Bicocca and was conducted according to the ethical standards of the Declaration of Helsinki (World Medical Organization, 1996). The general aim of the study and the procedure were explained to participants before obtaining informed consent; participants were informed that the experiment aimed to study body perception and that they would undergo one experimental session. At the end of 
the procedure, the specific scope of the study was also explained.

Data and analysis code are available on the Open Science Framework platform at the following link: https://osf.io/ 895nf/?view_only=b193f8145a6843adbdc2b18ea41726d2.

No part of the study procedures or analysis was preregistered prior to the research being conducted.

\section{Procedure}

Participants sat blindfolded on an armchair, with their arms behind their back. They underwent a Tactile Distance Task, modified from previous studies (Longo et al., 2015; Longo \& Golubova, 2017; Longo \& Haggard, 2012) to investigate a possible distortion of the metric representation of the leg.

Tactile Distance Task (TDT) A $3 \times 3$ grid of points (see Fig. 2) was stuck on the upper left leg. Before starting, we measured the length of the leg to fix the grid at the center of the leg. We considered the segment going from the hip (anterior part of the iliac crest) to the knee (inferior part of the kneecap). Adjacent points on the grid were separated by $5 \mathrm{~cm}$; row and column points ran along the medio-lateral and proximo-distal leg axes, respectively. On each trial, two locations were stimulated in sequence, with an inter-stimulus interval of approximately $1 \mathrm{~s}$. Tactile stimulations were manually applied using a knitting needle with a blunt end of $0.6 \mathrm{~mm}$ (size 6); the experimenter touched each point for about $1 \mathrm{~s}$. We used the same imaginary estimation proposed by Longo (2015), Longo and Morcom (2016): After each trial, participants verbally estimated the perceived distance between the two stimuli locations. There were 36 possible pairs of nine stimuli locations and two orders of presentation for each couple of points. For example, for the pair $\mathrm{AB}$, the experimenter could touch first point $\mathrm{A}$ and then point $\mathrm{B}$ ( $\mathrm{AB}$ order) or the other way around - first point $\mathrm{B}$ and then point $\mathrm{A}$ (BA order). The resulting number of trials was 72 pairs.

TDT - Global shape dissimilarity. For each participant, we constructed a symmetric matrix reflecting the pairwise perceived distances between pairs of points, with zeros on the diagonal (we averaged the distances expressed in the two orders of presentation of the same pair). We obtained the coordinates of each point of the grid, in a nine-dimensional space, by applying MDS to the distance matrix. Based on the eigenvalues of each dimension (see Electronic Supplementary Materials, Fig. 1), we extracted the first two dimensions of the solution, thus obtaining the coordinates in a bi-dimensional space. The first two dimensions accounted for a total of $56.15 \%$ of the variance on average (32.2\% first component; $23.96 \%$ second component).

Then, we reconstructed a perceptual configuration of the grid for each subject. To investigate the shape of the perceived grid, we generated multiple stretches of the real grid. We then identified which stretch minimized the Procrustes Distance to identify the shape that most likely corresponded to the perceptual grid. With this procedure, we can determine if the perceived grid is squared or rectangular and eventually the proportion of the sides of the rectangle.

To produce the stretched grids, we systematically changed the locations of the nine points multiplying the y-coordinates by a stretch parameter of between 0.33 (reducing the distance

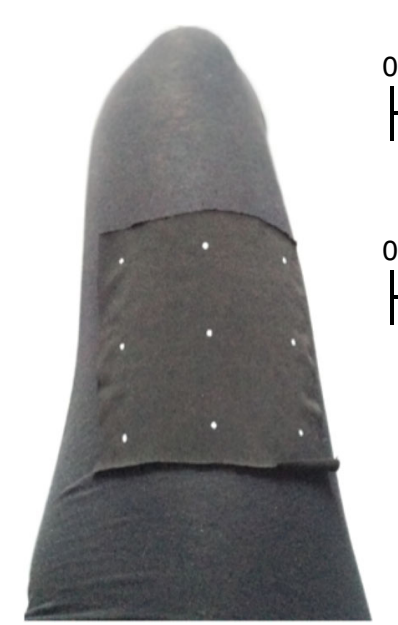

Fig. 2 Setting. The $3 \times 3$ grid of points $(10 \times 10 \mathrm{~cm})$ used to administer the stimuli for the body distance task was made of fabric and stuck on the upper left leg of the participant. On the right, the three response modalities
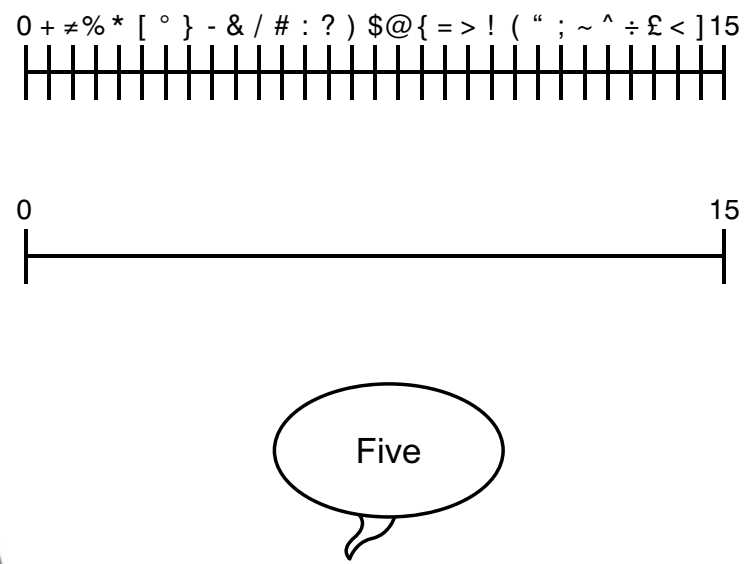

used in the main experiment are represented: the discrete line, the analogic line, and the verbal response 
of two points to one-third of the original distance) and 3 (three times the original distance) with a resolution of 0.0005 units in logarithmic space producing 4,415 different grids. When the stretch parameter is 1 the grid is squared; stretch parameters greater than 1 indicate a stretch in the proximo-distal axis, while stretch parameters less than 1 indicate a stretch in the medio-lateral axis (Longo \& Golubova, 2017).

We then run a Procrustes Alignment between the stretched grids and the participants' perceptive configurations. For each participant we thus had 4,415 indices of dissimilarity from the real grid of points, i.e., the Procrustes Distance (the square root of the residual sum of squared distances between pairs of homologous landmarks, which is not removed by PA). Procrustes Distance ranges between zero (the two configurations have the same shape) and one (the two configurations do not share spatial structure at all). We computed, for each subject, the stretch that minimized the Procrustes Distance to individuate which configuration is more similar to being perceived as one.

TDT-Misestimation. We compared the length estimated by the participants at each pair of stimuli to the real gap, and we calculated the percentage of perceived misestimation using the following equation: $\%$ misestimation $=$ (perceived distance - actual distance)/actual distance * 100. Since the misestimation additionally measures length judgment errors, independently of the shape of the grid, it represents a complementary index to the Procrustes Distance. Previous studies (Longo et al., 2015; Longo \& Morcom, 2016; Stone et al., 2018) have found different rates of distortion for distances running along the longitudinal (proximo-distal) or transversal (medio-lateral) axes. For this reason, we considered the direction of the tactile stimuli (medio-lateral axis/ proximo-distal axis), their distance (near $-5 \mathrm{~cm}$ like $\mathrm{AB}$ and $\mathrm{AD} / \mathrm{far}-10 \mathrm{~cm}$ like $\mathrm{AC}$ and $\mathrm{AG}$ ) and the order of presentation of the stimuli ( $\mathrm{AB}$ order/BA order) in the analysis of the misestimation.

\section{Analysis}

We conducted analyses with R 3.4.2 (R Development Core Team 2008) and JASP 0.8.4 (Jasp Team, 2017).

More specifically, to run MDS, we used the packages stats (cmdscale function); Procrustes Distance was calculated with the package vegan (function Procrustes). The misestimation percentage was calculated with an ad hoc formula in R. Model comparisons were run in $\mathrm{R}$, and we used the Akaike Information Criterion (AIC) and the loglik criteria to select the best fitting model. Bayesian statistics were run in JASP 0.8.4 (Jasp Team, 2017).
TDT - Global shape dissimilarity. We ran a Bayesian one-sample t-test on the stretch that minimized the Procrustes Distance, as implemented in Jasp 0.8.4. We compared the stretch to one, a value indicating a square grid. We adopted default parameters; namely, the estimation started from a non-informative prior with a Cauchy distribution with a location of 0 and a scale of 0.707 .

TDT-Misestimation. We performed a linear mixed model (LMM) selection with the misestimation as the dependent variable. Fixed effects tested were the Direction (medio-lateral axis/proximo-distal axis), the Distance (near/far), and the Order of the stimuli presentation (AB order/BA order). Model selection was performed implementing a null model with the only random factors as Subject (i.e., each participant). Then, a mixed-effect model was generated by adding fixed-effect factors and testing each model against the null one. Only fixed-effect factors that contributed to improving the model's fit were included in the final structure of the mixed-effect model. All model comparisons used the Chi-square test $(\alpha=$ .05 ). We then calculated the $95 \%$ CIs for the levels included in the final model, without running additional post hoc tests.

\section{Results}

TDT - Global shape dissimilarity. The Bayes factor $\left(\mathrm{BF}_{01}=2.87\right)$ showed anecdotal evidence in favor of the null hypothesis (average Stretch \pm StErr $=1.03 \pm$ $0.05)$. The analysis suggested that we did not have enough information to conclude if participants' perception of the tactile input reflects the shape of the original configuration of stimuli. Figure $3 \mathrm{~A}$ shows the difference between the real grid (dashed line) and the average of the subjects' perception (solid line).

TDT - Misestimation. We chose the more parsimonious model that contributed to improving the fit with the data. The model comparison reported in the Electronic Supplementary Material (Table 1) showed that the model that fits the data better included only Direction (medio-lateral axis/proximo-distal axis). Figure 3 shows, on the right, a higher underestimation for the proximo-distal distance (CI: -50.18; -14.75) than for the medio-lateral axis (CI: -42.30; -13.44).

\section{Short discussion}

Procrustes Distances did not show clear evidence in favor of the null or the alternative hypothesis, implying that our data 


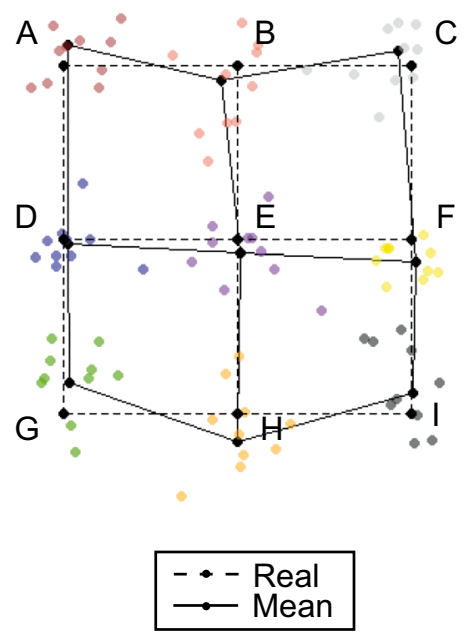

Fig. 3 Left: Results of the Multidimensional Scaling. The black dots connected by the solid line grid represent the mean of the subjects' perceptive responses compared with the actual grid (dashed line). Dots in different colors represent the perceived location of each point by each

were not informative about the shape of the tactile perception as compared to the real grid of stimuli. This result is not in line with previous studies about hand mapping (Longo et al., 2015; Longo \& Morcom, 2016) or with our results about specific distance judgments indicating a systematic distortion of tactile perception.

Indeed, when we calculated the percentage of perceived misestimation, all the estimated marginal means showed negative values, suggesting a general underestimation of the real distances. Interestingly, the model that fits the data better included only the variable Direction. This result indicates a higher underestimation for the proximo-distal length as compared to the medio-lateral one. Stone et al. (2018) found, similarly to the case of the hand, an underestimation in the proximo-distal direction, as the participants perceived the leg as being shorter than it is, a case that we replicated. Indeed, our results seem to go in the same direction as Stone's work with a more considerable reduction in proximo-distal distances rather than in the medio-lateral axis.

In the main experiment the sample size was increased and the experimental procedure was extended to other response methods.

\section{Main experiment - is Body Tactile Distance task affected by the response modality?}

We were interested in evaluating the possible biases emerging with specific cognitive processes related to different response modalities assessing their potential impact and the stability of the effect.

\section{Material and methods}

\section{Participants}

Twenty-four subjects participated in the study ( 23 female, mean age: $24.46 \pm 4.33$ years; mean school age: $16.42 \pm$ 1.56 years). All participants had normal or corrected-tonormal vision and were naïve to the purpose of the experiment; any of them participated in the preliminary investigation. All participants gave their written informed consent before participating. The study was approved by the local Ethics Committee "Commissione per la Valutazione della Ricerca, Dipartimento di Psicologia" of the University of MilanoBicocca and was conducted according to the ethical standards of the Declaration of Helsinki (World Medical Organization, 1996). The general aim of the study and the procedure were explained to participants before obtaining informed consent; participants were informed that the experiment was aimed at study body perception and that they would undergo two experimental sessions. At the end of the study, the specific scope of the study was explained.

\section{Procedure}

The experiment consisted of two sessions with 1 week of washout in between. During each session, participants underwent the body distance task, and they were asked to answer with different modalities.

Tactile Distance Task (TDT) Participants sat blindfolded on a chair, with their arms behind their back. They underwent the 
TDT following the same procedure as in the pilot experiment, but here we asked for estimates with three different types of judgments. Besides the verbal response (in $\mathrm{cm}$ ), which is supposed to be mediated by metric size awareness, we requested perceptual estimations, which is supposed to rely mostly on visuotactile integration. As we discussed, we tried to overcome the limitation of non-reproducible distances providing a visual reference for the perceptive recalibration.

We presented a reference line onto which the perceived distance was indicated. The line was presented on a computer screen with a constant orientation (i.e., horizontally). Because the visual stimulus may influence the judgment, we manipulated the reference line by providing it in two lengths $(15 \mathrm{~cm}$ and $30 \mathrm{~cm}$ ), both used in two ways (analogic or discrete). We asked participants to provide the distance estimation considering a range between $0 \mathrm{~cm}$ and $15 \mathrm{~cm}$, but we presented on a computer screen either a $15 \mathrm{~cm}$ or a $30 \mathrm{~cm}$ line. In the first condition, the reference length corresponded to the actual one presented $(15 \mathrm{~cm})$, while in the second condition, participants were asked to rescale the line $(30 \mathrm{~cm})$ to the reference length of $15 \mathrm{~cm}$, so that they had to double the perceived distance on the line. By doing so, we addressed the influence of a proportional rescaling. We hypothesized that if they used the line just as a response modality this additional cognitive process would not affect their estimations.

On the other hand, if they used the line as a real reference to represent the distance, we would expect a lower precision with the $30 \mathrm{~cm}$ line. Moreover, we were interested in evaluating if a discrete scale could improve participants' accuracy, as compared to an analogic one. On the analogic lines, for both the $15 \mathrm{~cm}$ and the $30 \mathrm{~cm}$ lengths, we provided only the start and end points $(0 \mathrm{~cm}$ and $15 \mathrm{~cm})$ without any other break or cue. Subjects were requested to point at the line at the corresponding perceived distance (analogic condition). To test the discrete scale, we provided participants with lines with breaks every $0.5 \mathrm{~cm}$ (in the case of the $15 \mathrm{~cm}$ line) or $1 \mathrm{~cm}$ (in the case of the $30 \mathrm{~cm}$ line). In order to avoid any explicit reference to numbers, only the extremities were indicated with $0 \mathrm{~cm}$ and $15 \mathrm{~cm}$, while the other 28 landmarks in between consisted of symbols (discrete condition). Participants were asked to verbally report the symbol corresponding to the perceived distance (Fig. 2). The rationale was that, by providing visual anchors for the judgment, participants would be more precise in reporting proportional differences than without anchors, resulting in a more accurate measure with less variability across individuals.

The experiment consisted of two sessions: in each session, we presented one of the two lengths $(15 \mathrm{~cm} / 30 \mathrm{~cm})$ for both the analogic and the discrete line, in a counterbalanced order. We also counterbalanced the order of presentation of the analogic and the discrete conditions during each session across participants. Only after the visual responses were participants asked to answer verbally (verbal condition). We administered the verbal condition either in the session that included the $15 \mathrm{~cm}$ lines or the one with the $30 \mathrm{~cm}$ lines, predicting no difference between the two sessions (i.e., no priming of the line length that precedes the verbal response).

This procedure resulted in six conditions that combined response modality and line length (Analogic $-15 \mathrm{~cm}$ line, Analogic $-30 \mathrm{~cm}$ line, Discrete $-15 \mathrm{~cm}$ line, Discrete $30 \mathrm{~cm}$ line, Verbal $-15 \mathrm{~cm}$ line, Verbal $-30 \mathrm{~cm}$ line).

We applied MDS to the distance matrix that emerged in each condition for each participant. We also computed a mean matrix, averaging all the responses given by each subject. Based on the eigenvalues (Electronic Supplemental Materials, Figs. 2 and 3), we reconstructed perceptual configurations of the grids in a bi-dimensional space. Table 1 reports the average variance explained by the first two dimensions in each condition. To compare the perceptual network of each participant and the real one, we followed the same procedure described for the preliminary experiment. Again, a value of 1 indicates a square-shaped grid; stretches greater than 1 indicate stretch in the proximo-distal axis, while stretches less than 1 indicate a stretch in the medio-lateral axis (Longo \& Golubova, 2017).

In addition, in all the conditions, we calculated the percentage of misestimation as in the preliminary experiment.

\section{Analysis}

We analyzed data with R 3.4.2 (R Development Core Team 2008) and JASP 0.8.4 (Jasp Team, 2017) utilizing the same software and packages of the preliminary experiment.

$T D T$ - Global shape dissimilarity. We conducted a Bayesian repeated-measures ANOVA with Response (Analogic/Discrete/Verbal) and Line Length (15/30) as

Table 1 Average variance explained by the first two dimensions in each condition. For each condition, we averaged the variance explained by the first two dimensions considering the entire sample of participants

\begin{tabular}{|c|c|c|c|c|c|c|c|}
\hline & $\begin{array}{l}\text { Analogic } \\
15 \mathrm{~cm}\end{array}$ & $\begin{array}{l}\text { Analogic } \\
30 \mathrm{~cm}\end{array}$ & $\begin{array}{l}\text { Discrete } \\
15 \mathrm{~cm}\end{array}$ & $\begin{array}{l}\text { Discrete } \\
30 \mathrm{~cm}\end{array}$ & $\begin{array}{l}\text { Verbal } \\
15 \mathrm{~cm}\end{array}$ & $\begin{array}{l}\text { Verbal } \\
30 \mathrm{~cm}\end{array}$ & $\begin{array}{l}\text { All conditions } \\
\text { averaged }\end{array}$ \\
\hline First dimension & $32.19 \%$ & $33.01 \%$ & $32.60 \%$ & $33.86 \%$ & $32,06 \%$ & $32.67 \%$ & $36.07 \%$ \\
\hline Second dimension & $24.18 \%$ & $25.18 \%$ & $24.77 \%$ & $25.73 \%$ & $24,81 \%$ & $24.93 \%$ & $27.35 \%$ \\
\hline Total & $56.37 \%$ & $58.19 \%$ & $57.37 \%$ & $59.59 \%$ & $56.87 \%$ & $57.61 \%$ & $63.42 \%$ \\
\hline
\end{tabular}


within-subjects factors, and the line presented in the first session $(15 / 30)$ as a between-subjects factor. We wanted to evaluate the replicability of the procedure and whether different testing modalities affected the shape of the perceptive grid. We performed a model selection, based on the Bayes factor, and we discussed the best fitting model. Given the result of the model selection, we also ran a Bayesian one-sample t-test on the mean stretch that minimized the Procrustes Distance. We compared the stretch to 1 , a value indicating a square grid. First, we started from a non-informative prior with a Cauchy distribution (location $=0$, scale $=0.707$ ). Then, we adopted an informative prior based on the result of the pilot experiment $(\mathrm{t}$ distribution: location $=0.132$, scale $=0.565$, degrees of freedom $=9$ ).

TDT - Misestimation. We also ran an additional model comparison with a linear mixed model (LMM) on the percentage of misestimation. We considered as possible fixed effects Session (I/II), Response (Analogic/Discrete/ Verbal), and Line length (15/30). We set participants as the random effect variable, and we calculated the $95 \%$ CIs for the levels of the factors that emerged in the best fitting model. Finally, we conducted a series of model comparisons between LMMs on the misestimation in each condition independently, as in the pilot experiment. Correlation analyses. We ran a series of Bayesian correlation analyses calculating Pearson's $r$ and the Bayes factor between the response conditions (averaging across $15 \mathrm{~cm}$ and $30 \mathrm{~cm}$ lines) and the line length (averaging

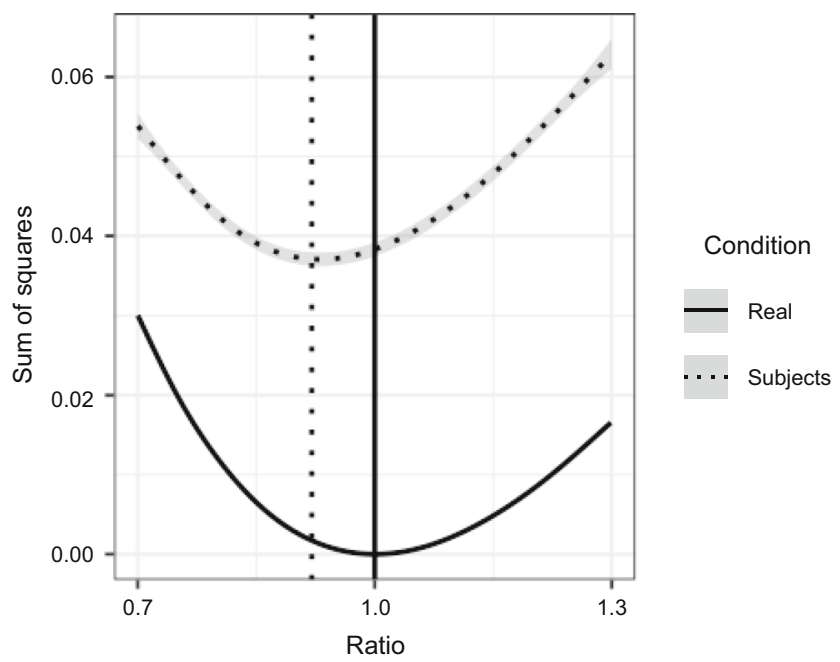

Fig. 4 Stretch that minimized the Procrustes Distance in the actual grid (solid line) and the mean of the subjects' perceptive responses (dotted line). The $y$-axis represents the sum of squares of the results from the Multidimensional Scaling; the $\mathrm{x}$-axis represents the stretch parameter with which we multiplicated the $\mathrm{x}$-coordinate across the type of response) to compare the similarity between the two indices (i.e., Global shape dissimilarity and Misestimation).

\section{Results}

TDT - Global shape dissimilarity. The model selection showed that the model that better fits the data is the null one, suggesting that our manipulations did not influence the global shape dissimilarity significantly (see the Electronic Supplementary Material Table 2 for a full report of model comparison). Since there is no difference between conditions, we reported the results for the averaged stretches that minimized the Procrustes Distance. Starting from a noninformative prior, the Bayes factors $\left(\mathrm{BF}_{10}=12.12\right)$ showed moderate to strong evidence in favor of the alternative hypothesis. When we considered the pilot result to set our informative prior, the Bayes factors $\left(\mathrm{BF}_{10}=13.18\right)$ confirmed moderate to strong evidence in favor of the alternative hypothesis, suggesting a difference between the real and the perceptive configurations of points (average Stretch \pm StErr $=0.93$ \pm 0.02 ). Figure 4 shows the differences between the stretch that minimized the Procrustes Distance in the actual grid (solid line) and the averaged subjects' responses (dotted line). A value lower than 1 indicates a stretch in the medio-lateral axis (Longo \& Golubova, 2017), meaning that distances between points in the proximo-distal axis were smaller than distances in the medio-lateral orientation.

TDT-Misestimation. The model selection reported in the Electronic Supplementary Materials (Table 3) shows that the best fitting model was the complete one, including Session, Response, and Line length. The full factorial model emerged as the best model, revealing significant effects of Session $(\mathrm{F}(1,10323)=241.38, \mathrm{p} \leq .001)$, Response $(\mathrm{F}(2,10323)=$ $262.19, \mathrm{p} \leq .001)$, and Line length $(\mathrm{F}(1,10323)=525.97, \mathrm{p}$ $\leq .001)$, and the interaction effects between Session and Response $(F(2,10323)=47.30, p \leq .001)$ and between Line length and Response $(F(2,10323)=90.05, p \leq .001)$. We also found a three-way interaction between Session, Line length, and Response $(\mathrm{F}(2,10323)=48.26, \mathrm{p} \leq .001)$, as shown in Fig. 5 (upper panel). Despite an overall underestimation of distances, responses provided on a $15 \mathrm{~cm}$ line were more accurate (CI: $-44.25 ;-21.45$ ), as compared with the $30 \mathrm{~cm}$ line (CI: -55.15; -32.07), independent of the type of line (analogic/ discrete). Moreover, in the first session, the verbal response seemed to be more precise (CI: $-37.19 ;-10.26)$, as compared with the analogic (CI: $-50,63 ;-29.33)$ and discrete responses (CI: -50.83; -29.23). In the second session, we identified almost the same pattern, except for the Verbal condition after 

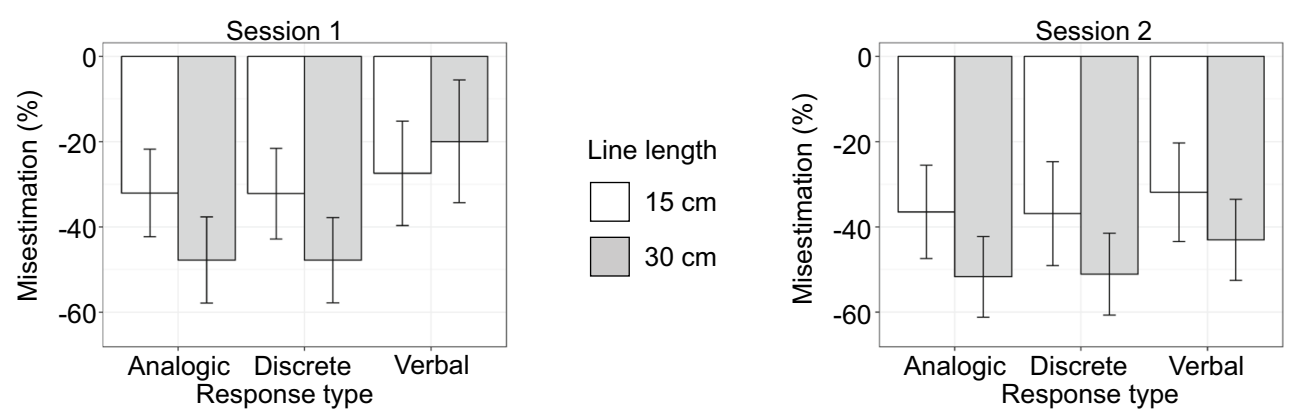

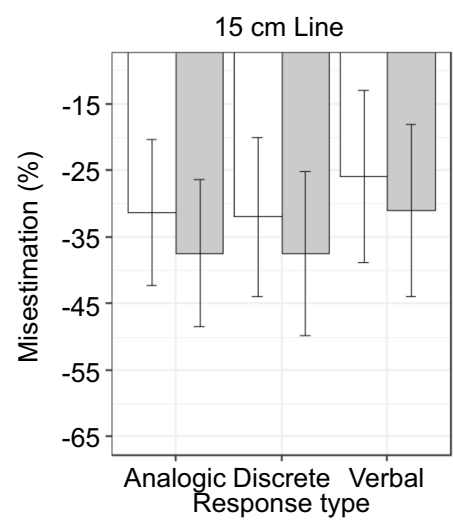

Fig. 5 First row: Best-fitting model resulting from the linear mixed model (LMM) selection with the misestimation as the dependent variable. The complete model included Session, Response, and Line length. The figure shows the percentage of misestimation in each response condition: Analogic, Discrete, and Verbal. The white columns represent the $15 \mathrm{~cm}-$ line condition, while the grey columns represent the $30 \mathrm{~cm}$-line condition. The more negative the misestimation values, the higher the underestimation of the leg length. Second row: Difference in misestimation between proximo-distal (grey columns) and medio-lateral axis (white columns).

the $30 \mathrm{~cm}$ line, which was found to have a larger underestimation of distances (CI: -52.61; -33.57).

Taking into account each condition separately, we considered significant effects emerged in each best fitting model (see Electronic Supplementary Materials, Table 4). We found a significant main effect of Direction in all conditions showing a greater reduction in proximo-distal distances compared to the medio-lateral axis (Analogic $15 \mathrm{~cm}$ line: Proximo-distal axis (CI: -48.47; -26.34); Medio-lateral axis (CI: -42.23; 20.31); Analogic $30 \mathrm{~cm}$ line: Proximo-distal axis (CI: 62.58; -43.55); Medio-lateral axis (CI: -58.55; -37.67); Discrete $15 \mathrm{~cm}$ line: Proximo-distal axis (CI: -49.79; 25.07); Medio-lateral axis (CI: -43.96; -19.95); Discrete $30 \mathrm{~cm}$ line: Proximo-distal axis (CI: -62.87; -43.98); Mediolateral axis (CI: -58.21; -37.44); Verbal $15 \mathrm{~cm}$ line: Proximodistal axis (CI: -43.95; -18.12); Medio-lateral axis (CI: -38.84; -12.89); Verbal $30 \mathrm{~cm}$ line: Proximo-distal axis (CI: -47.25; -
22.98); Medio-lateral axis (CI: -41.36; -11.83)), as shown in Fig. 5. Moreover, we detected a significant main effect of Distance in the Analogic $-15 \mathrm{~cm}$ line $(\mathrm{F}(1,834)=9.72, \mathrm{p} \leq$ $.05)$, Discrete $-15 \mathrm{~cm}$ line $(\mathrm{F}(1,838)=10.65, \mathrm{p} \leq .001)$, and Verbal $-30 \mathrm{~cm}$ line $(\mathrm{F}(1,838)=12.09, \mathrm{p} \leq .001)$. However, the direction of the effect was not clear, since we found a greater reduction for the short distances than for the bigger ones with the Analogic $-15 \mathrm{~cm}$ line and the Discrete $15 \mathrm{~cm}$ line, and the opposite pattern with the Verbal $-30 \mathrm{~cm}$ line. Finally, we found a significant interaction effect between Direction and Order with the Analogic $-15 \mathrm{~cm}$ line $(\mathrm{f}(1,834)$ $=8.46, \mathrm{p} \leq .05)$ and the Verbal $-15 \mathrm{~cm}$ line $(\mathrm{F}(1,837)=6.34$, $\mathrm{p} \leq .05)$.

Correlation analyses. Comparison between the two indices (i.e., Procrustes Distance and Misestimation) revealed anecdotal to moderate evidence in favor of the null 
Table 2 Bayesian correlations between the Procrustes Distance and the Misestimation in each response modality (averaging across $15 \mathrm{~cm}$ and $30 \mathrm{~cm}$ lines) and each line length (averaging across the type of response)

\begin{tabular}{|c|c|c|c|c|c|c|}
\hline & & pd_analogic & pd_discrete & pd_verbal & pd_ $15 \mathrm{~cm}$ & pd $\_30 \mathrm{~cm}$ \\
\hline \multirow{2}{*}{$\begin{array}{l}\text { misest } \\
\text { analogic }\end{array}$} & Pearson's r & 0,03 & 0,05 & 0,12 & 0,25 & $-0,15$ \\
\hline & $\mathrm{BF}_{01}$ & 3,92 & 3,84 & 3,43 & 2,06 & 3,19 \\
\hline \multirow[t]{2}{*}{ misest_discrete } & Pearson's r & 0,03 & 0,05 & 0,13 & 0,26 & 0,14 \\
\hline & $\mathrm{BF}_{01}$ & 3,92 & 3,87 & 3,33 & 1,99 & 3,19 \\
\hline \multirow[t]{2}{*}{ misest_verbal } & Pearson's r & 0,11 & 0,11 & 0,03 & 0,21 & $-0,11$ \\
\hline & $\mathrm{BF}_{01}$ & 3,48 & 3,48 & 3,92 & 2,49 & 3,46 \\
\hline \multirow[t]{2}{*}{ misest_15 cm } & Pearson's r & $-0,01$ & 0,08 & 0,19 & 0,32 & $-0,17$ \\
\hline & $\mathrm{BF}_{01}$ & 3,94 & 3,72 & 2,69 & 1,28 & 2,98 \\
\hline \multirow[t]{2}{*}{ misest_30 cm } & Pearson's r & 0,12 & 0,06 & $-0,02$ & 0,12 & $-0,09$ \\
\hline & $\mathrm{BF}_{01}$ & 3,42 & 3,82 & 3,93 & 3,37 & 3,66 \\
\hline
\end{tabular}

$p d$ indicates the stretch that minimized the Procrustes Distance, misest indicates Misestimation

hypothesis (Table 2). The fact that they do not correlate (or do so only marginally) suggests that the global shape dissimilarity and the direction bias convey different types of information.

\section{Discussion}

In order to evaluate if different testing modalities affected the shape of the perceptive grid, we performed a model selection based on the Bayes factor. The results suggest that our manipulations did not influence the global shape of the grids significantly. Since there is no difference between conditions, we considered the averaged responses given by each participant, and we ran a Bayesian one-sample ttest on the mean stretch that minimized the Procrustes Distance. We compared the stretch to 1 , the value indicating a square grid. Results were not substantially affected by the use of a non-informative prior or the use of the results of the preliminary experiment as prior. Results showed a difference between a square grid and the configuration of points emerged in participants' responses. In particular, the stretch that minimized the Procrustes Distance was lower than 1, confirming that distances between points in the proximodistal axis were smaller than distances in the medio-lateral orientation. Importantly, because Procrustes alignment normalizes the sizes, Longo and Golubova (2017) specified that a stretch applied to the medio-lateral axis produces that same result to the inverse stretch applied to the proximodistal axis (i.e., doubling one side produces the same result as halving the other one). Thus, this method cannot indicate which specific axis is responsible for the distortion (if there is one). Either way, these results strongly support the idea that humans have a distorted map of the body in perceiving tactile events (Longo et al., 2015; Longo \& Morcom, 2016; Stone et al., 2018), which seems to be resistant to contextual experimental effects.

Notably, previous studies have found a stretch along the medio-lateral (transversal) axis, and an equivalent compression of the proximo-distal (longitudinal) one (Longo et al., 2015; Longo \& Haggard, 2011; Longo \& Morcom, 2016; Stone et al., 2018). We checked for the presence of this systematic bias, calculating the percentage of distance error and evaluating any influence of the points' orientation. Results showed that there was a general underestimation of the tactile distances; moreover, this distortion was higher on the proximo-distal axis than on the medio-lateral one. Regardless of the response method, subjects perceived a general reduction of the leg sizes, and, in particular, a contraction of the leg length, in line with the literature (Longo et al., 2015; Longo \& Haggard, 2011; Longo \& Morcom, 2016; Stone et al., 2018). The resistance of the distortion to stimulus information confirmed the results found by Ambroziak, Tamè, and Longo (2018). The authors found distal biases in knuckle localization when participants were localizing their knuckles both on an empty silhouette of the hand and on a photograph. They concluded that such mislocalizations reflect conceptual misrepresentations of hand structure.

With regard to the response modality, we compared verbal and perceptive responses, considering that the former could be based on the metric size awareness necessary to provide an imaginary estimation. At the same time, the latter could be mediated by perceptual recalibration, thus influenced by visuotactile integration, and therefore they may differ. In addition, we modulated the size of the line used to answer, and its scale (continuous or discrete). We assessed the influence of the response modality on both shape and distance perception through the Procrustes Distances and the percentage of 
misestimation. The Bayesian repeated-measures ANOVA on the Procrustes Distance showed that the best fitting model was the null one, suggesting that the global shape of the perceptive grid is independent of the response type, and it is a replicable methodology.

Nevertheless, considering the direction bias, the LMM revealed an interaction effect between Session, Response modality, and Line Length. Besides the overall underestimation of distances, we found that in the first session, verbal responses were the most accurate compared to analogic and discrete ones. It is possible that the perceptive "cue" of the line was a confounding factor for distance judgments.

In both perceptual conditions, we expected participants to compare the distance between the tactile stimuli with the line presented. Since it requires a more complex cognitive process, the visual stimulus could influence subjects' estimations. In the case of the verbal response, subjects were only asked to say the number corresponding to the distance they perceived. Comparison with the line on the screen needed a subsequent transformation that could introduce more noise; the findings of the line length support this hypothesis. When the visual line did not correspond to the length represented, the bias was emphasized: responses provided on a $30 \mathrm{~cm}$ line showed a greater underestimation. In this case, we introduced an additional cognitive step: the line had to be scaled to the length that was represented. This result seems to relate to a well-known distance perception bias since longer distances are usually misestimated as compared to shorter distances both on the body and in space (Longo \& Golubova, 2017; Longo \& Morcom, 2016; Plumert, Kearney, Cremer, \& Recker, 2005). In this case, it was not the distance that was judged to be longer, but the reference used to answer. With regard to the scale used in the analogic and discrete conditions, we did not find any difference. This result suggests that the perceptive cues provided with the discrete line did not help participants' responses. In the second session, we found almost the same pattern except for the verbal responses given after the $30 \mathrm{~cm}$ line, where we found a higher underestimation of distances. We do not have any specific suggestion about this result; we limit our observation by now considering that it might also be a simple regression-toward-the-mean effect.

The Bayesian correlation analysis between the Procrustes Distance and Misestimation revealed anecdotal to moderate evidence in favor of the null hypothesis, suggesting that the two indices are unrelated, and so provide different information. We propose that the two scores should be used synergistically and not as two mutually exclusive alternatives.

\section{General discussion}

We used a modified version of the tactile distance task proposed by Longo (Longo \& Golubova, 2017; Longo \&
Haggard, 2012; Longo et al., 2015) in order to test body distance perception on the leg. We evaluated the reliability of the method, if it is replicable, and how much it is resistant to potential cognitive confounds, thus providing support for its initial scope of being a method for perceptual measure of body-metric representation. Additionally, we provided a robust methodological validation to the shape dissimilarity index (i.e., the Procrustes Distance) and the directional bias (i.e., the misestimation score), two different approaches of estimating perceptual biases in the tactile distance task. We first assessed the feasibility of this specific task on the upper leg of 10 healthy subjects to pilot the procedure and the timing needed for the experiment, and then we explored the potential biases in the estimation procedure in a new, independent, fullsample experiment.

In the pilot experiment, we did not found a distortion of subjects' perceptual maps as compared to the actual grid. It is of note that in assessing the particular distance judgements, the results highlighted a higher underestimation for the proximo-distal length as compared to the medio-lateral one.

In the main experiment, we evaluated the possible influences of different kinds of response modalities that have been used in previous studies, such as imaginary verbal estimates and perceptive recalibration (Longo \& Haggard, 2011; Longo et al., 2015; Longo \& Morcom, 2016; Stone et al., 2018). As we pointed out in the introduction, perceptive recalibration might be limited by non-reproducible distances. To overcome this limitation, we provided a visual reference (i.e., a line) modifying its length and the scale used. We questioned if scaling the real distance using a proportional reproduction would be as successful as providing a copy of the physical range and if it is better to employ an analogic or a discrete line with perceptive cues. We found a distortion of the tactile distances on the leg, characterized by an overall underestimation and a more specific compression of the proximo-distal axis, confirming previous studies on both the hand and the leg (Green, 1982; Longo et al., 2015; Longo \& Haggard, 2011; Longo \& Morcom, 2016; Stone et al., 2018). Longo and Haggard (2011) have hypothesized that estimates made in the longitudinal (proximo-distal) direction are underestimated more than in the transverse direction due to the shape of the tactile receptive fields (RFs) on the dorsum of the hand. They proposed a pixel model for afferent input coding, based on the anisotropy of the RFs. It is known that RFs are oval-shaped in the dorsum of the hand, with the long axis running proximodistally and the short one running mediolaterally (Alloway, Rosenthal, \& Burton, 1989; Brown, Fuchs, \& Tapper, 1975). The same receptive locations on the skin would be mapped into circular pixels that comprised the body model (a stored representation of the metric properties of the body). If tactile distance perception is determined by the number of RFs between touched stimuli, medio-lateral distances will appear larger then proximo-distal ones, because of the 
higher number of RFs. This hypothesis explains why the hand is represented as being wider than it is. Recently, Stone and collaborators (2018) used a similar task on the leg and found a good estimation on the transverse dimension but again an underestimation on the longitudinal (proximo-distal) direction. These findings are in line with the previous work by Green (1982), which showed that participants significantly underestimated distances applied on their lower limbs in the proximo-distal direction compared to the transverse one. We have not been able to find a reference about the RFs shape on the legs. In speculating, until any better hypothesis is proposed, we must assume that the pixel hypothesis is correct. If so, the studies by Green (1982), Stone et al. (2018), and the present work are compatible with RFs also being oval-shaped on the legs. Future studies could directly address this issue. From a behavioral point of view, we can conclude that the perception of the leg as based on tactile input appears to be distorted with regard to its physical dimensions (Stone et al., 2018), similar to the dorsum of the hand.

Another critical point, which Longo and Golubova (2017) characterized, is the curvature of the hand (convex on the dorsum; concave on the palm), which results in points along the medio-lateral axis being slightly closer in a projection onto a plane then they really are. This raises the point that there are two ways in which the distance between two stimulated points on the skin might be perceived. First, the distance could be thought of in terms of distance along the surface of the skin. Second, the distance could be thought of in terms of the 3-D Euclidean distance of the shortest path between the stimulated points (Longo \& Golubova, 2017). After the task, we asked our participants for the strategy that they used in distance estimation. We did not quantify their responses, but thanks to their feedback, we can hypothesize the existence of two groups. Part of the sample thought of distance in 3-D Euclidean space, as suggested in their responses: "I traced a line between the touched points"; "I imagined the abstract representation of distance." Another group of participants thought of distance on the skin surface: "I visualized the distance on the leg"; "I imagined the convex shape of the leg and a meter upon it." It is our opinion that participants could think of distance both on the skin surface and in 3-D Euclidean space, due to personal strategies.

The Procrustes Distance and the percentage of misestimation differed in evaluating the response modalities. Indeed, even if they referred to the same task, they represent slightly different information, confirming the results of our correlation analysis. The Procrustes Distance provides a dissimilarity index between the global shape of two configurations, without discriminating the type of distortion. It considers the global shape of each grid, but it cannot distinguish between a wide and short configuration and a tall and tight one. On the contrary, the percentage of perceived misestimation compares the distance provided by the participants at each stimulus pair and the actual gap. This measure is independent of the shape and focuses on the distances between each point of the grid. Our results suggest that different response modalities do not affect the perception of the general form of our body parts. The characteristic distortion of the grid with a higher reduction of distance estimation along the proximo-distal axis, as compared to the medio-lateral one, is maintained regardless of the specific task demands. This similarity is not valid if we focus on directional biases: the integration between tactile inputs and visual stimuli influences estimate precision. Indeed, verbal responses were more accurate. To understand the different results, we need to distinguish between a holistic point of view, in the case of Procrustes Distances, and a more specific one, in the case of distance misestimation. We propose that our brain preserves the perception of the general shape of our body parts, although distorted, independently according to the specific demand. However, if we examine the particular distance perception, we can notice a dependence of the cognitive processes and the sensory inputs involved. Such an organization would allow maintaining coherence and continuity in our body experience.

In conclusion, we confirmed the TDT as a suitable method in the characterization of the body metric (Longo, 2015; Tamè et al., 2019). It turned out to be a reliable and replicable method producing consistent results applicable to different body parts. The global shape dissimilarity was shown to be particularly resistant to contextual experimental factors. However, it did not convey absolute values and directional information.

The directional bias was partially affected by the response modality used to report the estimates.

Our results suggest that the verbal response is the most precise method to reveal lower directional biases. Finally, by directly comparing the two scoring indices, we provided substantial support to the combined use of the two indices as they give complementary information about body metric representation distortions.

Acknowledgements We thank Clara Paschino for her contribution in data collection.

\section{Compliance with ethical standards}

All data and materials for all experiments are available at: https://osf.io/ 895nf/?view_only=b193f8145a6843adbdc2b18ea41726d2

Conflict of interest The authors declare that they have no conflicts of interest.

\section{References}

Alloway, K. D., Rosenthal, P., \& Burton, H. (1989). Quantitative measurements of receptive field changes during antagonism of GABAergic transmission in primary somatosensory cortex of cats. Experimental Brain Research, 78(3), 514-532. https://doi.org/10. 1007/BF00230239 
Ambroziak, K. B., Tamè, L., \& Longo, M. R. (2018). Conceptual distortions of hand structure are robust to changes in stimulus information. Consciousness and Cognition, 61, 107-116.

Bookstein, F. L. (1991). Morphometric tools for landmark data. Cambridge: Cambridge University Press.

Brown, P. B., Fuchs, J. L., \& Tapper, D. N. (1975). Parametric studies of dorsal horn neurons responding to tactile stimulation. Journal of Neurophysiology, 38(1), 19-25. https://doi.org/10.1152/jn.1975. 38.1.19

Carroll, J. D., \& Arabie, P. (1998). Multidimensional scaling. In Measurement, judgment and decision making (pp. 179-250). Academic Press.

Cox, T. F. (2001). Multidimensional scaling used in multivariate statistical process control. Journal of Applied Statistics, 28(3-4), 365-378.

Friston, K. J., Frith, C. D., Fletcher, P., Liddle, P. F., \& Frackowiak, R. S. J. (1996). Functional topography: Multidimensional scaling and functional connectivity in the brain. Cerebral Cortex, 6(2), 156164.

Green, B. G. (1982). The perception of distance and location for dual tactile pressures. Perception \& Psychophysics, 31(4), 315-323. https://doi.org/10.3758/BF03202654

Goodall, C. (1991). Procrustes methods in the statistical analysis of shape. Journal of the Royal Statistical Society: Series B (Methodological), 53(2), 285-321.

Longo, M. R. (2015). Implicit and explicit body representations. European Psychologist, 20(1), 6-15. https://doi.org/10.1027/10169040/a000198

Longo, M. R., \& Golubova, O. (2017). Mapping the internal geometry of tactile space. Journal of Experimental Psychology: Human Perception and Performance, 43(10), 1815-1827. https://doi.org/ 10.1037/xhp0000434

Longo, M. R., \& Haggard, P. (2010). An implicit body representation underlying human position sense. Proceedings of the National Academy of Sciences, 107(26), 11727-11732.

Longo, M. R., \& Haggard, P. (2011). Weber's illusion and body shape: Anisotropy of tactile size perception on the hand. Journal of Experimental Psychology: Human Perception and Performance, 37(3), 720-726. https://doi.org/10.1037/a0021921

Longo, M. R., \& Haggard, P. (2012). A 2.5-D representation of the human hand. Journal of Experimental Psychology: Human Perception and Performance, 38(1), 9-13. https://doi.org/10.1037/ a0025428

Longo, M. R., Azañón, E., \& Haggard, P. (2010). More than skin deep: Body representation beyond primary somatosensory cortex. Neuropsychologia, 48(3), 655-668.

Longo, M. R., Mancini, F., \& Haggard, P. (2015). Implicit body representations and tactile spatial remapping. Acta Psychologica, 160, 77-87. https://doi.org/10.1016/j.actpsy.2015.07.002
Longo, M. R., \& Morcom, R. (2016). No Correlation between Distorted Body Representations Underlying Tactile Distance Perception and Position Sense. Frontiers in Human Neuroscience, 10(November), 1-8. https://doi.org/10.3389/fnhum.2016.00593

Olatunji, B. O., Kim, S. K., \& Wall, D. (2015). Extracting body image symptom dimensions among eating disorder patients: The Profile Analysis via Multidimensional Scaling (PAMS) approach. Body Image, 15, 16-23.

Plumert, J. M., Kearney, J. K., Cremer, J. F., \& Recker, K. (2005). Distance perception in real and virtual environments. $A C M$ Transactions on Applied Perception, 2(3), 216-233. https://doi. org/10.1145/1077399.1077402

Rohlf, F. J., \& Slice, D. (1990). Extensions of the Procrustes method for the optimal superimposition of landmarks. Systematic Biology, $39(1), 40-59$.

Sadibolova, R., Tamè, L., Walsh, E., \& Longo, M. R. (2018). Mind the gap: The effects of temporal and spatial separation in localization of dual touches on the hand. Frontiers in Human Neuroscience, 12, 55.

Stone, K. D., Keizer, A., \& Dijkerman, H. C. (2018). The influence of vision, touch, and proprioception on body representation of the lower limbs. Acta Psychologica, 185(February), 22-32. https://doi.org/ 10.1016/j.actpsy.2018.01.007

Tamè, L., Braun, C., Holmes, N. P., Farnè, A., \& Pavani, F. (2016). Bilateral representations of touch in the primary somatosensory cortex. Cognitive Neuropsychology, 33(1-2), 48-66.

Tamè, L., Azañón, E., \& Longo, M. R. (2019). A Conceptual Model of Tactile Processing across Body Features of Size, Shape, Side, and Spatial Location. Frontiers in Psychology, 10(February). https://doi. org/10.3389/fpsyg.2019.00291

Team, J. A. S. P. (2017). JASP (Version 0.8.4) [Computer software].

Team, R. C. (2008). R development core team: An introduction to R: Notes on R, A programming environment for data analysis and graphics (electronic edition, 2008), also by WN Venables and DM Smith.

Tsakiris, M. (2010). My body in the brain: A neurocognitive model of body-ownership. Neuropsychologia, 48(3), 703-712.

Widiger, T. A., Trull, T. J., Hurt, S. W., Clarkin, J., \& Frances, A. (1987). A multidimensional scaling of the DSM-III personality disorders. Archives of General Psychiatry, 44(6), 557-563.

World Medical Organization. (1996). Declaration of Helsinki. British Medical Journal, 313(7070), 1448-1449.

Publisher's note Springer Nature remains neutral with regard to jurisdictional claims in published maps and institutional affiliations. 